\title{
De la problématique des articles synopsis dans la compilation des dictionnaires au Gabon*
}

\author{
Gilles Saphou-Bivigat(13788647@sun.ac.za / saphoubivigat@yahoo.fr), et \\ Edgard Maillard Ella(13751727@sun.ac.za / ellamaillard@yahoo.fr), \\ Département d'afrikaans et hollandais, Université de Stellenbosch, \\ Stellenbosch, Republique d'Afrique du Sud
}

\begin{abstract}
Le caractère multilingue et multiculturel du Gabon résulte de l'environnement linguistique et sociolinguistique, dont il faut tenir compte dans la compilation des dictionnaires. Par rapport aux articles simples les articles synopsis offrent une meilleure approche dans le transfert des données linguistiques et culturelles dans les dictionnaires. Autrement dit, au Gabon l'attention doit porter sur l'utilisation des articles synopsis dans la compilation des dictionnaires, en vue de refléter dans ses ouvrages la diversité linguistique et culturelle de ce pays. Ceci est d'autant plus vrai que l'utilisation des articles synopsis ne dépend aucunement de la typologie du dictionnaire.

Cet article à pour but de suggérer les différentes approches méthodologiques à partir desquelles les lexicographes et compilateurs des dictionnaires peuvent utiliser les articles synopsis pour présenter les données linguistiques et sociolinguistiques. Ces approches méthodologiques prennent en compte l'utilisation de ces articles dans différents types des dictionnaires. Ces développements seront discutés et évalués en fonction de leurs capacités à traiter l'aspect encyclopédique des items lexicaux. L'accent ne sera pas seulement porté sur le traitement des articles synopsis dans la nomenclature, mais aussi sur les post-textes qui peuvent aussi contenir un texte avec une liste de tous les items qui sont les unités de traitement de ces articles dans cette partie centrale du dictionnaire.

En d'autres termes, le point central de cet article est l'utilisation des articles synopsis dans la nomenclature, complétée par les régistres alphabétiques contenus dans les post-textes en vue de la compilation des différents types des dictionnaires polyaccessibles en fournissant aux usagers des moyens d'accès rapide pour atteindre les données aussi bien linguistiques que culturelles.
\end{abstract}

Mots-clés: ARTICLE SYNOPSIS, ITEMS LEXICAUX, ARTICLE SIMPLE, SIGNE LEMME, ASPECTS LINGUISTIQUES, TYPES DE MACROSTRUCTURES, ASPECTS LINGUISTIQUES ET EXTRALINGUISTIQUES, ITEMS LEXICAUX CENTRÉS SUR LA CULTURE

Abstract: The Problematics of Synopsis Articles in the Compilation of Dictionaries in Gabon. Gabon has a multilingual and multicultural character resulting in a

* Cet article a été presenté comme une communication à la Neuvième Conférence Internationale Annuelle de l'Association Africaine pour la Lexicographie (AFRILEX), organisée par le Groupe de Recherche en Langues et Cultures Orales (GRELACO), Université Omar Bongo, Libreville, Gabon, 18-23 juillet 2004.

Lexikos 16 (AFRILEX-reeks/series 16: 2006): 270-279 
linguistic and sociolinguistic environment that must be taken into account in the compilation of dictionaries. Compared to simple articles, synopsis articles offer a better approach to the transfer of linguistic and cultural information in dictionaries. In other words, in Gabon, the focus should be on the use of synopsis articles in dictionaries that must be compiled to take into account and convey in these works the linguistic and cultural diversity of this country. This is very important because the utilisation of synopsis articles does not depend on dictionary typology.

The aim of this article is to suggest different methodological approaches for which lexicographers and compilers of dictionaries can use synopsis articles to present linguistic and cultural data. These methodological approaches take into account the use of these articles in different types of dictionaries. These developments will be discussed and evaluated for their ability to present and treat the encyclopedic aspect of lexical items. The emphasis will not only be on the treatment of synopsis articles in the central list, but also on back matter texts that can also contain a text with a list of all the items which are the treatment units of these articles in this central part of the dictionary.

In other words, the focal point of this article is directed at the level of the utilisation of synopsis articles in the central list which will be completed by alphabetical registers in the back matter texts for the compilation of different types of dictionaries which will be polyaccessible in offering to users rapid access means to reach both linguistic and cultural data.

Keywords: SYNOPSIS ARTICLE, TYPES OF MACROSTRUCTURES, SINGLE ARTICLE, LEMMA SIGN, LINGUISTIC AND EXTRALINGUISTIC ASPECTS, TARGET GROUP, DATA CATEGORIES, CULTURE-BOUND LEXICAL ITEMS

\section{Introduction}

L'un des débats actuels dans les discussions théoriques sur les problèmes lexicographiques met l'accent sur la diversité culturelle et linguistique. Ce débat conduit inéluctablement à l'utilisation des articles synopsis qui garantissent le transfert des données aussi bien linguistiques que culturelles. Dans la recherche métalexicographique actuelle, on distingue les articles simples et les articles synopsis. Les articles simples sont ceux qui présentent le traitement type assigné au signe lemme moyen, c'est-à-dire un traitement linguistique. Quant aux articles synopsis, ils offrent au-delà du traitement linguistique un surcroît de catégorie des données d'une nature encyclopédique permettant d'améliorer le transfert des données.

Par ailleurs, chaque dictionnaire doit être compilé selon les spécificités culturelles et linguistiques de la communauté à laquelle il est destiné. Dans une société multilingue et multiculturelle comme le Gabon, les dictionnaires jouent un rôle social non négligeable dans l'échange et le transfert des connaissances. Comme référence, on citera Wiegand (1997) et Gouws (1999) qui sont favorables à une attention particulière à l'usage des articles synopsis dans les dictionnaires pour une société multilingue et multiculturelle, où ces ouvrages de référence ont en filigrane un rôle de bâtisseur de la nation et de responsabilité sociale. Dans cette lignée d'idées Mavoungou (2002) a déjà mis en relief l'im- 
portance de ce type d'articles dans la compilation des dictionnaires incluant les langues gabonaises qui sont des véhicules de cultures très diversifiées.

Ces différentes perspectives attribuées au rôle et au but véritable du dictionnaire auront définitivement des implications dans la compilation des dictionnaires au Gabon en incluant des défis intéressants qui font face non seulement aux lexicographes mais aussi aux linguistes, dans cet environnement multilingue et multiculturel que représente le Gabon.

\section{Orientation théorique et méthodologique de l'utilisation des articles synopsis}

\subsection{Caractéristiques des usagers des dictionnaires gabonais}

Chaque dictionnaire doit être compilé selon les besoins spécifiques d'un groupe d'usagers bien déterminé. Par conséquent, la compilation de n'importe quel dictionnaire exige auparavant l'identification des usagers indiqués, leurs besoins et habiletés lexicographiques. Par conséquent, la sélection de la macrostructure ainsi que le traitement microstructural des lemmes comme la structure d'adressage doivent être déterminés par le profil de l'usager cible. Compte tenu du rôle et du but véritable du dictionnaire dans un cadre de vulgarisation et d'unification des connaissances et d'un environnement multiculturel et multilingue, les besoins spécifiques ainsi que les demandes et habiletés des usagers de provenances diverses doivent être pris en compte dans la planification et la compilation des dictionnaires au Gabon.

De même, les usagers cibles des dictionnaires au Gabon représentent une communauté linguistique et culturelle hétérogène. À part des locuteurs des langues gabonaises il faut aussi prendre en compte les autres communautés étrangères qui voudraient avoir accès à ces langues gabonaises. Les lexicographes ne peuvent pas satisfaire tous ces usagers sur une base égale. Leurs décisions par rapport à la planification et la structure des dictionnaires au Gabon doivent être guidées par le profil sociolinguistique du prototype de l'usager cible. Les lexicographes des dictionnaires gabonais doivent tenir compte des usagers cibles dans leurs divergences et convergences culturelles aussi bien que des points de vue idéologiques. Le dictionnaire ne devrait pas prendre une position impartiale. La sélection macrostructurale aussi bien que le traitement des lemmes doivent faire preuve d'une attitude neutre quant à la culture et quant aux idéologies de certains items lexicaux. L'influence de la culture sur le sens d'un mot peut se refléter sur plusieurs items lexicaux comme père par exemple:

Les sens des mots père et mère varieront dans les langues gabonaises selon que l'on est dans une communauté à tradition matrilinéaire ou dans une communauté à tradition patrilinéaire. C'est ainsi que dans une communauté à tradition matrilinéaire telle que les Vili dans la Nyanga la 
mère, nommée par l'unique item lexical «mame» [mâ:mə] fera référence à la génitrice mais aussi aux oncles maternels qui jouent le même rôle et ont la même autorité que la mère génitrice. Or dans une communauté à tradition patrilinéaire comme celle des Fang, le père nommé par l'unique item lexical «tara» [tárà], fera référence au géniteur, mais il se référera aussi aux oncles paternels qui jouent le même rôle et ont la même autorité que le père géniteur.

\subsection{Les articles synopsis dans la macrostructure des dictionnaires gabonais}

La compilation des dictionnaires gabonais rendra les langues gabonaises plus accessibles parmi les différentes communautés et jouera un rôle majeur dans la promotion des langues gabonaises parmi les communautés linguistiques étrangères. Ceci a des implications dans l'organisation de la macro- et microstructure et met l'accent sur le besoin de clarté et d'usage facile des structures d'accès. D'un point de vue macrostructural, il est important que la sélection du lemme soit en fonction des critères de sélection avec l'usage des fréquences comme facteur dominant, c'est-à-dire en se basant sur les listes des fréquences, des transcriptions des interviews, de l'usage dans la presse, des programmes de télévision, etc. pour aboutir à la sélection d'items lexicaux constituant le noyau du vocabulaire.

\subsection{Les articles synopsis dans la microstructure des dictionnaires gabonais}

Le traitement microstructural de chaque lemme a pour but de rendre l'usager apte à obtenir les compétences nécessaires pour l'usage pratique du mot en communication réelle. Ainsi, lorsque la macrostructure offre à l'usager l'accès à une certaine partie du vocabulaire, la microstructure offre l'accès aux caractéristiques linguistiques de ces mots. Dans la situation du Gabon, la microstructure devrait aussi offrir un accès aux caractéristiques culturelles de ces mots. Cet accès devrait être traité d'une manière non ambiguë en prévision du transfert le plus facile possible des données. La simplicité dans la définition est un concept largement respecté en vue de maximiser les chances de l'usager de comprendre les définitions des mots. À cet effet, la notion de grande simplicité devrait être un principe majeur dans l'utilisation des articles synopsis.

\section{Orientation théorique et méthodologique pour le traitement de l'accès des usagers aux données}

\subsection{Les articles synopsis et l'utilisation des structures d'accès}

Les dictionnaires ne véhiculent pas seulement les données à travers les signes linguistiques et codes mais aussi par l'usage des conventions ou signes typo- 
graphiques. Ces codes ou marqueurs typographiques ont pour but de contribuer au transfert optimal des données. Malheureusement, comme on peut souvent constater, une mauvaise utilisation ou une abondance de ces marqueurs et codes peuvent rendre plus complexes l'accès à la microstructure et peuvent empêcher par cela une bonne interprétation du texte lexicographique. L'usager type des dictionnaires gabonais sera un usager qui ne sera pas encore familier avec l'utilisation des dictionnaires particuliers des langues gabonaises, ainsi qu'avec l'utilisation du dictionnaire en général. Or, cet usager comptera sur ces dictionnaires pour une assistance spontanée sans pour autant être obligé de suivre les renvois d'articles dans les autres composantes du dictionnaire, surtout lorsque ces renvois ne sont pas codés et nécessitent encore d'être decodés pour avoir l'aide souhaitée.

En incluant systématiquement les articles synopsis, les dictionnaires gabonais devraient répondre aux besoins d'un accès instantané de l'information en mettant en place des structures d'accès efficace. Pour cela l'utilisation des structures d'accès doit être faite avec la plus grande simplicité. Cette complémentarité devrait aboutir à diminuer les marqueurs typographiques ainsi que les autres entrées qui rendent l'article trop complexe. Cette combinaison devrait aussi introduire des nouvelles conventions visant à rendre moins ambigu l'accès à chaque catégorie des données contenue dans l'article. Par exemple, la façon dont les indications tonales ont été élaborées dans le dictionnaire fangfrançais/français-fang, œuvre du révérend Samuel Galley (1919-1959), pourrait être simplement marquée par la représentation phonétique du lemme dans la compilation de nouveaux dictionnaires incluant la langue fang ou n'importe quelle autre langue gabonaise. En effet, Galley a noté, par une lettre entre parenthèse l'indication relative à la tonalité du lemme. Pour le mot $a b a$, il se présente comme suit:

ABA (h) n. 4, pl. meba (dialecte atsi). Corps de garde où se tiennent les hommes. Syn.: abeñy (h).

Dans cet article le (h) indique une haute tonalité. Ceci est confus dans la mesure où il n'y a aucune indication quant à la voyelle portant le ton, et représente un accès assez difficile pour les usagers qui doivent s'astreindre à maîtriser cette structure d'accès assez complexe parmi tant d'autres. Le lexicographe ferait mieux de donner l'indication tonale dans la représentation phonétique du lemme ABA dont la première voyelle a le ton haut, et la deuxième une voyelle longue. On pourrait ainsi avoir:

ABA, [ába:] ...

En d'autres termes, dans la compilation des dictionnaires gabonais qui impliqueraient l'utilisation des articles synopsis, les lexicographes devraient limiter les structures d'accès dans la mesure où leur utilisation abondante pourraient aboutir à la confusion chez les utilisateurs. 


\subsection{La recherche d'une plus grande clarté}

Comme cela a été mentionné plus haut l'utilisation des renvois internes d'articles devraient coïncider avec l'objectif d'atteindre une plus grande clarté. Une caractéristique dominante des dictionnaires gabonais qui inclurait les articles synopsis devrait être le clair et explicite accès à travers lequel les données sont transférées. Cela implique donc que l'accès devrait être facilité par des entrées qui offrent un transfert direct des données. L'application de ces principes doit amener à reconsidérer l'utilisation des abréviations comme entrées microstructurales, c'est-à-dire que non seulement le transfert codé des données grammaticales devrait être reconsidéré, mais devrait être aussi valable pour l'usage dans plusieurs dictionnaires des abréviations des classes des mots. Par exemple, au lieu des entrées:

$$
\text { «afan ( } p l \text { me } \phi-) \text {, [áfàn] qui signifie forêt }
$$

le lexicographe fera mieux de donner une présentation plus claire et plus explicite de la forme du pluriel:

$$
\text { «afan ( } p l \text { mefan)». }
$$

La plus grande clarté, combinée à une meilleure chance d'accéder aux données, peut être obtenue en abandonnant le système d'indication des données morphologiques, comme les pluriels, les diminutifs et les temps passés en notant seulement le suffixe qui s'y applique, au lieu de l'entrée entière.

Ce genre d'approche rend l'usager apte à obtenir directement et instantanément l'accès à l'information dont il/elle a besoin avec moins de chance de mal interpréter le système d'accès dans le dictionnaire.

\subsection{Contrôle de la densité des données dans l'utilisation des articles sy- nopsis}

Simplicité et clarté devraient être considérées comme deux points majeurs du succès des transferts des données dans l'utilisation des articles synopsis. Quand bien même ces deux aspects peuvent être véhiculés à travers les structures des renvois internes des articles, les lexicographes des dictionnaires gabonais pourraient aussi bien utiliser d'autres méthodes pour atteindre cet objectif. Cette méthode est d'évaluer et de réguler la densité des données appropriées à chaque dictionnaire. Selon Hausmann (1989: 982, cité par Gouws 1993: 39) la densité des données d'un dictionnaire est la relation quantitative entre la macro- et microstructure. Une augmentation des données dans la microstructure d'un dictionnaire, sans pour autant en changer la macrostructure impliquerait donc une augmentation des données de cette macrostructure. Ainsi, un dictionnaire pourrait être orienté soit de façon macro- ou microstructurale ou pourrait également maintenir un équilibre des données entre la macro- et microstructure. 
Les restrictions d'espace dans le traitement lexicographique peuvent être résolues par le transfert implicite des données. Un exemple de ce transfert implicite des données dans un dictionnaire général bilingue à dimension trilingue peut être la présentation d'un registre d'équivalents dans les posttextes.

Ainsi, dans un dictionnaire général bilingue à dimension trilingue vili/ français/anglais, le transfert implicite des données trilingues pourrait être la présentation d'un registre d'équivalents vili/français/anglais dans les posttextes.

\subsection{Orientation bidirectionnelle dans l'utilisation des articles synopsis}

L'orientation bidirectionnelle dans l'utilisation des articles synopsis interviendra à priori dans la compilation des dictionnaires bilingues. Ainsi, vu la densité des données dans l'utilisation des articles synopsis, les lexicographes des dictionnaires gabonais devraient maintenir un équilibre entre la macro- et microstructure en adoptant une orientation bidirectionnelle selon la conception de Hausmann et Werner (1991: 2740, cité par Gouws 2001: 118) qui est relative à la notion du dictionnaire biscopal.

Dans l'utilisation des articles synopsis, les lexicographes devraient mettre l'accent sur la délimitation de la microstructure de telle façon que l'accès à l'information ne soit pas obstrué par une grande quantité de données. En prenant les décisions sur la densité des données à inclure dans un dictionnaire spécifique, les lexicographes devraient encore une fois prendre en considération les besoins et les aptitudes du groupe cible. En d'autres termes, la densité des données à inclure dans les articles synopsis dépend du type et du véritable but du dictionnaire à compiler, ainsi que des caractéristiques, des besoins et des problèmes du groupe cible, c'est-à-dire l'ensemble des potentiels des usagers pour lesquels un dictionnaire est destiné. Le véritable but du dictionnaire est en terme métalexicographique l'objectif visé par la compilation d'un dictionnaire de transférer, au moyen de données lexicologiques, les données concernant l'ensemble d'items lexicaux qui sont inclus comme unités du traitement pour assurer l'acquisition par le pouvoir linguistique de l'utilisateur cible (cf. Gouws 2001: 103).

\section{Pour une approche concise et simplifiée de la macrostructure}

Dans un Gabon multilingue et multiculturel l'usager type des dictionnaires gabonais sera premièrement intéressé par l'orthographe et la définition des lemmes ainsi que leur utilisation en situation de communication. Ainsi ces ouvrages de références destinés à vulgariser et à standardiser les langues gabonaises doivent fournir une variété de catégories de données telle que la prononciation, la morphologie et l'étymologie nécessaires. Néanmoins, cela ne signifie pas qu'il faut inclure tous les aspects sémantiques d'un mot mais plutôt de 
fournir les données nécessaires en vue de rendre ce mot accessible, compréhensible pour la plus grande variété possible des usagers. En effet, à cause de l'insuffisance des connaissances linguistiques et culturelles liées aux langues gabonaises, le souci premier des usagers des dictionnaires gabonais sera d'avoir à leur portée des dictionnaires d'accès faciles qui leurs permettront d'avoir l'information utile et nécessaire pour la définition et l'usage correct des items lexicaux en langues gabonaises, c'est-à-dire que ces dictionnaires doivent être des outils fonctionnels en ce qu'ils doivent contribuer à parfaire les aptitudes des usagers à communiquer en langues gabonaises.

Toutefois, il est également important de mentionner ici la relation entre le type du dictionnaire à compiler et la façon dont seront utilisés les articles synopsis. En effet, la quantité des données à inclure dans ces articles lorsqu'ils sont destinés à un dictionnaire encyclopédique ne sera pas la même que lorsque ceux-ci sont destinés à un dictionnaire pédagogique scolaire par exemple.

\subsection{Les exemples scripturaux}

Baunebjerg Hansen (1990: 19, cité par Gouws 1993: 42) considère les exemples scripturaux comme une catégorie lexicographique à part. Comme le soutient Rademeyer (1992, cité par Gouws 1993: 42) dans le même ordre d'idées, les exemples scripturaux peuvent avoir une variété de fonctions dans un dictionnaire. L'utilisation des exemples scripturaux se révèlera donc en premier lieu dans le contexte de l'utilisation des articles synopsis qui contiennent entre autres des données culturelles qui doivent être comprises par les usagers. En effet, les exemples scripturaux ont cette fonction pragmatique dans la réception et la production des textes, en éclairant et en distinguant par exemple les différents sens polysémiques d'un lemme, qui permettent une utilisation performante des items lexicaux dans un contexte de communication.

\subsection{Les exemples picturaux}

Les exemples picturaux, même s'ils sont souvent assez utilisés, sont malheureusement parfois considérés comme redondants et comme des ornements aux articles du dictionnaire. Toutefois, ces exemples ont une grande valeur lexicographique, puisque tout en étant un complément à la définition verbale, ils ajoutent une information ou une explication supplémentaire. Par cela, ils permettent d'améliorer la compréhension de l'usager cible. L'inclusion des exemples picturaux dans les articles synopsis ne se limitera pas seulement, comme il est de coutume, aux seuls dictionnaires monolingues. Les exemples picturaux devraient être pris en compte dans la compilation de n'importe quel type de dictionnaire incluant les langues gabonaises dès que le besoin se ferait sentir. Leur usage jouerait un rôle encyclopédique non négligeable dans la compréhension des données extralinguistiques inclues dans les articles synopsis. Ainsi, 
ils ne seront pas redondants car ils contribueront à un transfert optimal des données nécessaires aux besoins et aux demandes des usagers des dictionnaires de langues gabonaises.

Par exemple, le lemme désignant mbandja doit être précédé de l'illustration malgré la définition. En effet, ce lemme pourrait être défini comme une «sorte de hangar» ou "petite maison» qui sont deux concepts qu'un usager européen par exemple aurait du mal à représenter car le mbandja représente toute autre chose en réalité qu'un hangar ou une petite maison selon la conception européenne.

\section{Conclusion}

Les articles synopsis constituent des moyens efficaces lorsqu'il s'agit de véhiculer les données linguistiques et extralinguistiques dans un dictionnaire. Leur traitement doit reposer sur une base théorique et pédagogique du point de vue lexicographique et sociologique, mais aussi sur des principes linguistiques. Dans un Gabon multilingue et multiculturel les dictionnaires de langues gabonaises doivent offrir un accès non seulement à ces différentes langues mais aussi aux différentes cultures véhiculées par ces langues. Ces dictionnaires, en incluant les articles synopsis, pourraient donc non seulement servir à relier les écarts linguistiques et culturels entre les communautés linguistiques du Gabon, mais pourraient également permettre aux locuteurs natifs des langues gabonaises de rendre performante leurs compétences à communiquer dans leurs propres langues.

\section{Références bibliographiques}

Baunebjerg Hansen, G. 1990. Artikelstruktur im zweisprachigen Wörterbuch. Überlegungen zur Darbietung von Übersetzungsäquivalenten im Wörterbuchartikel. Lexicographica. Series Maior 35. Tübingen: Max Niemeyer.

Galley, S. 1964. Dictionnaire fang-français et français-fang, suivi d'une grammaire fang. Neuchâtel: Henri Messeillier.

Gouws, R.H. 1993. Afrikaans Learner's Dictionaries for a Multilingual South Africa. Lexikos 3: 2948.

Gouws, R.H. 1999. Die maatskaplike gerigtheid van die metaleksikografie in 'n meertalige samelewing. Cours d'ouverture. Stellenbosch: Universiteit van Stellenbosch Drukkery.

Gouws, R.H. 2001. Formation lexicographique: approches et thèmes. Emejulu, J.D. (Éd.). 2001. Éléments de lexicographie gabonaise. Tome I: 95-134. New York: Jimacs-Hillman.

Hausmann, F.J. 1989. Das Definitionswörterbuch. Hausmann, F.J. et al. (Éds). 1989-1991: 981-988.

Hausmann, F.J. et R.O. Werner. 1991. Spezifische Bauteile und Strukturen zweisprachiger Wörterbücher: Eine Übersicht. Hausmann, F.J. et al. (Éds.). 1989-1991: 2729-2769.

Hausmann, F.J. et al. (Éds). 1989-1991. Wörterbücher: Ein internationales Handbuch zur Lexikographie/ Dictionaries: An International Encyclopedia of Lexicography/Dictionnaires: Encyclopédie interna- 
tionale de lexicographie. Handbücher zur Sprach- und Kommunikationswissenschaft 5.1-5.3. Berlin/New York: Walter de Gruyter.

Mavoungou, P.A. 2002. Synopsis Articles in the Planning of a Trilingual Dictionary: YilumbuFrench-English. Lexikos 12: 181-200.

Rademeyer, L. 1992. Die funksies van verbale voorbeeldmateriaal in eentalige woordeboeke. M.A. thèse inédite. Stellenbosch: Université de Stellenbosch.

Wiegand, H.E. 1997. Die gesellschaftliche Verantwortung der wissenschaftliche Lexikographie. Hermes, Journal of Linguistics 18: 177-202. 